OPEN

SUBJECT AREAS:

MATERIALS SCIENCE

STRUCTURAL PROPERTIES

Received

1 September 2014

Accepted

30 September 2014

Published

16 October 2014

Correspondence and requests for materials should be addressed to N.L. (nanli@lanl.gov) orJ.W. (wangj6@lanl.

gov)

\section{In situ Nanoindentation Study of Plastic Co-deformation in Al-TiN Nanocomposites}

\author{
N. Li ${ }^{1}$, H. Wang ${ }^{2}$, A. Misra ${ }^{1,3}$ \& J. Wang ${ }^{2}$
}

\begin{abstract}
'Materials Physics and Applications Division, MPA-CINT, Los Alamos National Laboratory, Los Alamos, New Mexico 87545, USA,
${ }^{2}$ Materials Science and Technology Division, MST-8, Los Alamos National Laboratory, Los Alamos, New Mexico 87545, USA,

${ }^{3}$ Department of Materials Science and Engineering, University of Michigan, Ann Arbor, Michigan 48109, USA.
\end{abstract}

We performed in situ indentation in a transmission electron microscope on Al-TiN multilayers with individual layer thicknesses of $50 \mathrm{~nm}, 5 \mathrm{~nm}$ and $2.7 \mathrm{~nm}$ to explore the effect of length scales on the plastic co-deformability of a metal and a ceramic. At $50 \mathrm{~nm}$, plasticity was confined to the Al layers with easy initiation of cracks in the TiN layers. At $5 \mathrm{~nm}$ and below, cracking in TiN was suppressed and post mortem measurements indicated a reduction in layer thickness in both layers. The results demonstrate the profound size effect in enhancing plastic co-deformability in nanoscale metal-ceramic multilayers.

T he enhancement of plastic deformability in ceramics will enable the design of novel composites with high hardness and measurable ductility that are used in energy and defense related technologies and aerospace engineering ${ }^{1-3}$. Metal/ceramic multilayers have come into greater focus due to their promising mechanical, physical and chemical properties, making them practically useful for harsh environments and extreme loading ${ }^{3}$. These composites show improvement in hardness, toughness, wear resistance, thermal resistance, shock resistance and irradiation resistance, to name a few. Compared to metals, ceramics are lacking in room temperature plasticity and fracture toughness ${ }^{4-5}$, because of a high lattice friction stress on most slip systems at low homologous temperatures. In the competition between dislocation glide and brittle fracture in ceramics at low homologous temperatures, brittle fracture wins since the stresses necessary to activate dislocation glide are significantly higher than fracture stress. However, localized dislocation activity in ceramics at room temperature has been observed underneath the indenter. Oden et al. ${ }^{6}$ and Minor et al. ${ }^{7}$ reported evidence for dislocation motion in the TiN indentation tests by transmission electron microscopy (TEM) analysis of the post-indentation microstructure, implying localized plasticity under hydrostatic constraint in indentation. Localized plasticity has also been reported in cementite $\left(\mathrm{Fe}_{3} \mathrm{C}\right)$ lamellae in pearlitic steels ${ }^{8}$ under wire drawing that also involves imposed hydrostatic pressure. More recent investigations have indicated that under severe plastic deformation, decomposition of $\mathrm{Fe}_{3} \mathrm{C}$ at cementite/ferrite interface occurs that releases $\mathrm{C}$ into the ferrite matrix and may account for the thickness reduction in cementite lamellae.

Metal/ceramic layered composites have been fabricated and extensively tested under various types of loading including compression ${ }^{9-13}$, tension ${ }^{14}$ and nanoindentation ${ }^{15-18}$ normal to the interface. Most of these studies show that the metallic layer undergoes plastic deformation while the ceramic layer remains in the elastic regime until it fractures. However, as the bilayer period is reduced to a few nanometers, plastic co-deformability was inferred in Al-TiN multilayers from compressive stress-strain curves ${ }^{19,20}$. In this investigation, we have performed in situ indentation in a transmission electron microscope (TEM) on Al-TiN multilayers with various layer thicknesses, $50 \mathrm{~nm}, 5 \mathrm{~nm}$ and $2.7 \mathrm{~nm}$ to explore the effect of reduced layer thickness on metal-ceramic plastic codeformability.

\section{Results}

Figure 1 shows two cross-sectional TEM images of the as-deposited films with the individual layer thickness $50 \mathrm{~nm}$ and $5 \mathrm{~nm}$. The diffraction patterns (DPs) confirm the orientation relation between the $\mathrm{Al}$ and TiN layers, (111) $\mathrm{Al}||(111) \mathrm{TiN} \|$ interface and $\left.\langle 110\rangle_{\mathrm{Al}} \|<110\right\rangle_{\mathrm{TiN}}$, and the growth direction is along [111]. The DP in Fig. $1 \mathrm{~b}$ is consistent with epitaxial growth of $\mathrm{Al}$ and $\mathrm{TiN}$ within a column. The multilayered films have a columnar structure with a $<111>$ fiber texture. In the plane of the film, the individual columns with a preferred $<111>$ 


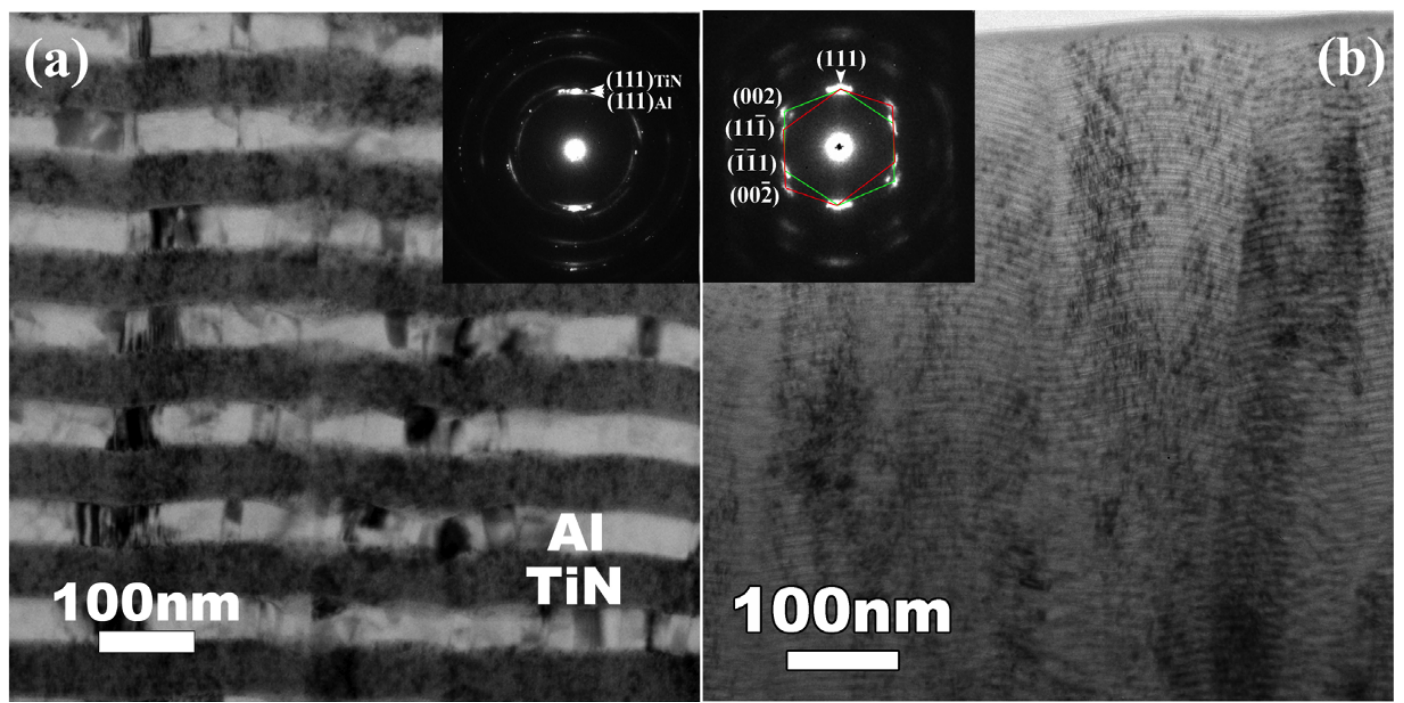

Figure 1 TEM images of the as-deposited films with the individual layer thickness (a) $50 \mathrm{~nm}$ and (b) $5 \mathrm{~nm}$.

growth direction may be misoriented with respect to each other by $60^{\circ}$ resulting in the formation of $\Sigma 3\{112\}$ incoherent twin boundaries (ITBs) between adjoining columns (Fig. 2 and Supplementary Figures S1-S3).

Four TEM images of the $50 \mathrm{~nm} \mathrm{Al-50} \mathrm{nm} \mathrm{TiN} \mathrm{multilayer} \mathrm{during}$ in situ indentation in Fig. 2 show the thickness reduction in the Al layers and cracks in the first TiN layer (see Supplementary Movie I). The Al layers in one column are polycrystalline (Fig. 2a), containing multiple $\Sigma 3\{112\}$ ITBs. The first Al layer beneath the indenter experiences significant plastic deformation, and the thickness reduces from $45 \mathrm{~nm}$ to $37 \mathrm{~nm}$ to $27 \mathrm{~nm}$ (Fig. 2a-c). High-resolution TEM (HRTEM) images in Fig. 3a and 3b clearly show the initial AlTiN interface with the (111) plane and the deformed Al-TiN interface that is a low angle tilt boundary with the tilt angle of 9.4 degrees. The formation of the tilt boundary is ascribed to the pileup of the gliding dislocations in the Al layer at the interface. In addition, we also observed the migration of $\Sigma 3\{112\}$ ITBs in the Al layers (see Supplementary Figure $\mathrm{S} 1)^{21}$. In the TiN layers, there is no detectable thickness reduction (Fig. 2). The first crack initiates in the TiN layer from the Al-TiN interface (Figs. $2 \mathrm{c}$ and $3 \mathrm{~b}$ ). This is a mode I crack due to the tensile stress resulting from the TiN bending underneath the indenter tip. The second crack initiates in the TiN layer from the top surface (Fig. 2d and Fig. 3c). According to the crystallography of the TiN layer, the second crack surface is close to a $\{111\}$ plane (Fig. 3c). It is also noticed that the Al layer near the second crack does not fracture associated with the opening of the crack (Fig. 3d). We studied the stress state associated with the crack initiation using finite element method and found that both the cracks are initiated by the tensile stress (see Supplementary Figure S4).

Figure 4 shows several TEM images of the $5 \mathrm{~nm} \mathrm{Al-5} \mathrm{nm} \mathrm{TiN}$ multilayer during in situ indentation (see Supplementary Movie II). The thickness reduction in both the Al layers and the TiN layers were observed. The first and second Al layers experience a significant plastic deformation beneath the indenter. The thickness of the second Al layer reduces from $\sim 5.5 \mathrm{~nm}$ to $\sim 1.9$ associated with the bending of the first TiN layer (Figs. $4 \mathrm{a}$ and $4 \mathrm{~b}$ ). The HRTEM image in Fig. 4c shows a tilt boundary that is associated with the high density of dislocations in the Al layer and at the Al-TiN interface. The first TiN layer experiences the obvious bending, the local radius beneath the indenter decreases from the initial infinity to the range of $102 \sim 156 \mathrm{~nm}$ (Fig. 4b). A slight thickness reduction in the first TiN layer is evidenced in the HRTEM images (Figs. 4d and 4e), from $5.87 \mathrm{~nm}$ to $5.02 \mathrm{~nm}$, the reduction corresponds to three atomic layers. We also characterized one lattice dislocation in the TiN layer that is identified by the Burgers circuit (Fig. 4f), implying plastic deformation in the TiN layer associated with the dislocations motion. To confirm the plastic deformation in TiN layers associated with dislocations, we performed another indentation test with a small displacement (see the Supplementary Figure S2). The local compressive strain is estimated to be $-8 \%$ according to the thickness reduction of the first five layers (from $29.2 \mathrm{~nm}$ to $26.9 \mathrm{~nm}$ ). Two important features are observed. $\Sigma 3\{112\}$ ITBs are present in the pristine TiN layers. Under compression, the $\mathrm{ITB}_{1}$ migrates for $2 \mathrm{~nm}$ (see Supplementary Figure S2-a and S2-b) and the $\mathrm{ITB}_{2}$ dissociates into two tilt boundaries bounded with a 9R phase with a width of $4.5 \mathrm{~nm}^{21}$ (see Supplementary Figure S2-c and S2-d). According to crystallography of TiN, $\Sigma 3\{112\}$ ITBs can be described as an array of Shockley partial dislocations with the net zero Burgers vector within an unit of three $\{111\}$ planes $^{21,22}$. Our DFT calculation shows that a full dislocation $1 / 2<110>\{111\}$ can dissociate into two Shockley partial dislocations $1 / 6<112>\{111\}$ that are bounded with a stacking fault of $1.0 \sim 1.5 \mathrm{~nm}$ under zero shear stresses, implying that a Shockley partial dislocation can glide on the $\{111\}$ plane accompanying with the creation of the intrinsic stacking fault ${ }^{23,24}$. Thus, the shear stresses that are generated due to the bending of the TiN layer favor the migration and/or dissociation of an ITB ${ }^{25,26}$. More importantly, we again captured a mobile lattice dislocation in the TiN layer (Fig. S2-e), which is observed initially in the layer in Fig. S2a and finally glides into the interface in Fig. S2-b. Experimental observation $^{6-8}$ and our DFT calculations ${ }^{23}$ suggest the preferred slip system $1 / 2<1-10>\{110\}$ in the TiN at room temperature. However, the e-beam direction is along the $\langle 110\rangle$ in our TEM characterization, we can only characterize one component of the $1 / 2<1-$ $10>\{110\}$ dislocation, i.e., one extra $\{111\}$ plane. Thus, we believe that the dislocation identified in our HRTEM images is belonging to the slip system $1 / 2<1-10>\{110\}$.

Figure 5 shows the TEM images of the $2.7 \mathrm{~nm} \mathrm{Al-2.7} \mathrm{nm}$ TiN multilayer during in situ indentation (also see the Supplementary Movie III). Fig. 5c, a HRTEM image, shows a highly coherent structure between the $\mathrm{Al}$ and TiN layers. Due to the small lattice difference and the fine layer thickness, the layered structures in Figs. 5a, 5b, 5d, and $5 \mathrm{e}$ can be only characterized with the bi-layer thickness. During the film growth, $\mathrm{Al}$ atoms have a high diffusivity on $\mathrm{Al}$ (111) surface. This facilitates the epitaxial growth of a flat Al layer. Correspondingly, the TiN layer grows on the flat Al surface, forming a sharp and flat $\mathrm{Al}-\mathrm{TiN}$ interface. The coherent strains in the $\mathrm{Al}$ and TiN layers result in a sharp contrast in the TEM image. However, the low diffusivity in the TiN layer may result in a relatively rough $\mathrm{TiN}$ 

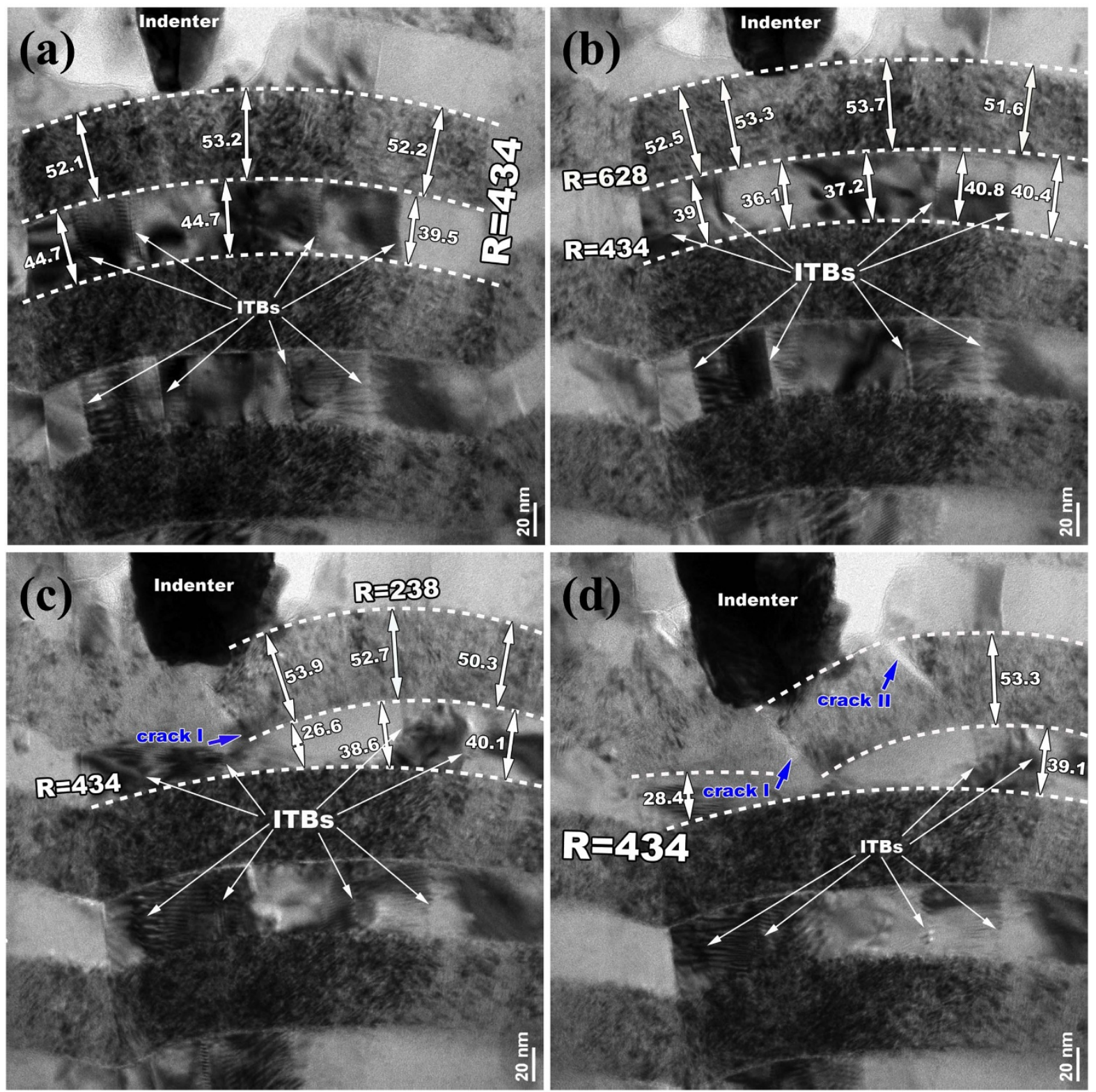

Figure 2 TEM images of the $50 \mathrm{~nm} \mathrm{Al-50} \mathrm{nm} \mathrm{TiN} \mathrm{multilayer} \mathrm{during} \mathrm{in} \mathrm{situ} \mathrm{indentation,} \mathrm{showing} \mathrm{the} \mathrm{thickness} \mathrm{reduction} \mathrm{in} \mathrm{the} \mathrm{Al} \mathrm{layers} \mathrm{and} \mathrm{cracks}$ in the first TiN layer. $\Sigma 3\{112\}$ ITBs are present in the Al layers and migrate during indentation.

(111) surface. The newly formed $\mathrm{TiN}-\mathrm{Al}$ interface is relatively rough, reducing the contrast in terms of the coherent strain. Thus, the sharp Al-TiN interface benefits us to distinguish a bi-layer thickness (Fig. 5c). Different from the thick layers, the first 5 bi-layers beneath the indenter experience a significant thickness reduction and no crack was observed. Fig. $5 \mathrm{~d}$ and $5 \mathrm{e}$ show the thickness reduction in the first three bi-layers from $17.5 \mathrm{~nm}$ to $6.3 \mathrm{~nm}$, corresponding to a strain of $-64 \%$.

\section{Discussion}

Our experiments revealed a profound size effect on the mechanical deformation of Al-TiN multilayers, i.e., significant plastic codeformation is only observed when the layer thickness is reduced to a few nanometers. In the multilayers with $50 \mathrm{~nm}$ layer thickness, no layer thickness reduction was measured in TiN. The first Al layer below the indenter experiences a significant plastic deformation due to the bending of the first TiN layer directly beneath the indenter, as a result, the initial Al-TiN (111) interface changes into a low angle tilt interface associated with the accumulation and the pileup of the dislocations in the $\mathrm{Al}$ layer. The second $\mathrm{TiN}$ layer remains flat, indicating the low compressive stress acting on the second TiN layer. This could be ascribed to the low mechanical strength of the thick Al layer. When the layer thickness decreases to $5 \mathrm{~nm}$, the first three AlTiN bi-layers experience slight elastic-plastic deformation, in particular, the plastic deformation in the TiN layers was characterized with the evidence of the lattice dislocations in the TiN layers. When the layer thickness decreases to $2.7 \mathrm{~nm}$, the first five Al-TiN bi-layers beneath the indenter tip experience a significant elastic-plastic deformation, $\sim 60 \%$ compressive strain without detectable cracks, while retaining the interface plane orientation (111)Al $\mid(111) \mathrm{TiN}$ consistent with compatible plasticity between the $\mathrm{Al}$ and TiN layers.

The plastic co-deformation of metal-ceramic multilayers thus can be rationalized as follows. Plastic deformation first commences in metal layers because of the relatively lower yield strength and higher mobility of dislocations as compared to ceramics. Dislocations glide in the metal layers confined by metal-ceramic interfaces, depositing dislocations at the interfaces. The glide dislocations from the metal layer deposited at the interfaces represent the plastic incompatibility between the two layers. The stress field of the interface dislocation 

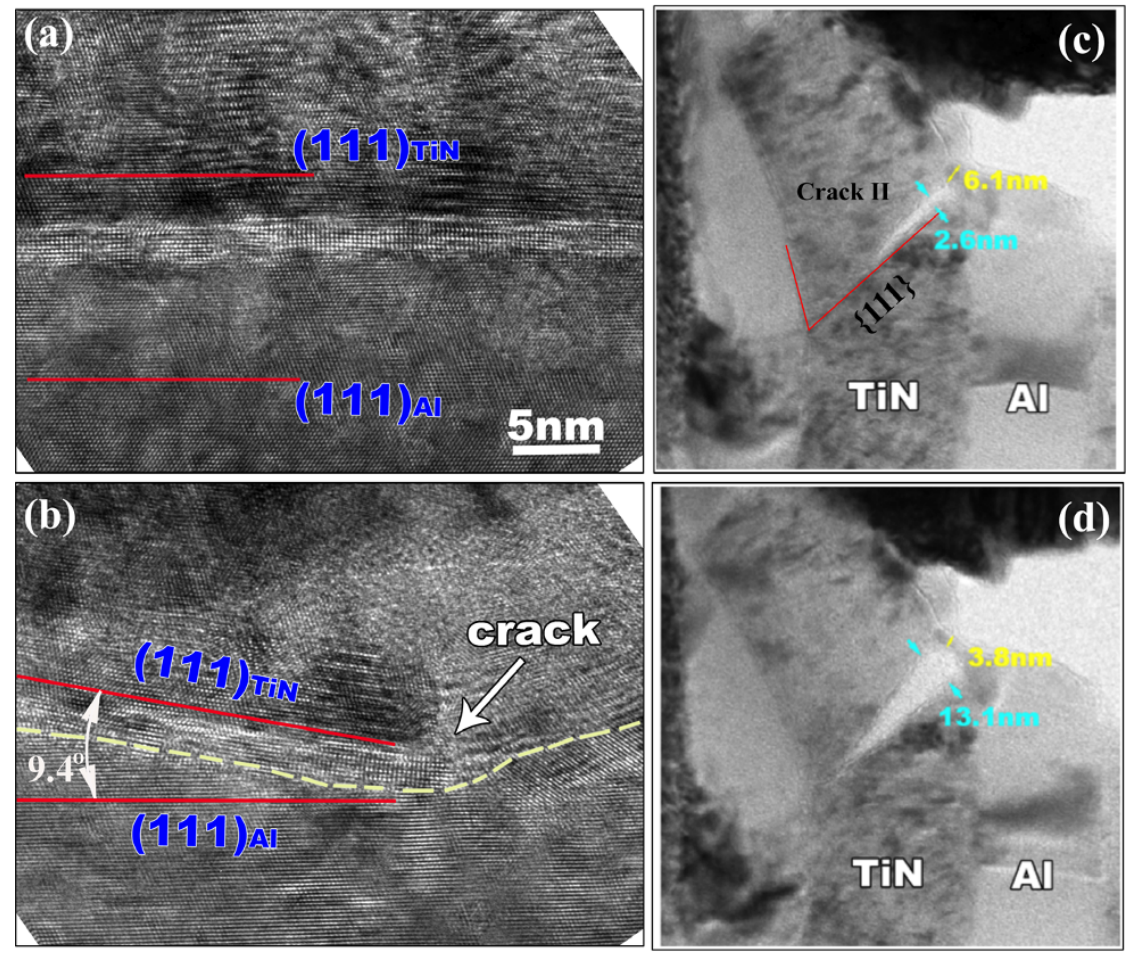

Figure $3 \mid$ HRTEM images show the initial Al-TiN interface (a) and the deformed Al-TiN interface (b). The red lines indicate the (111) plane.

(c) and (d) TEM images show the initiation and propagation of the crack II in the first TiN layer. The Al layer near the crack reduces the thickness from $6.1 \mathrm{~nm}$ to $3.8 \mathrm{~nm}$ without fracture.
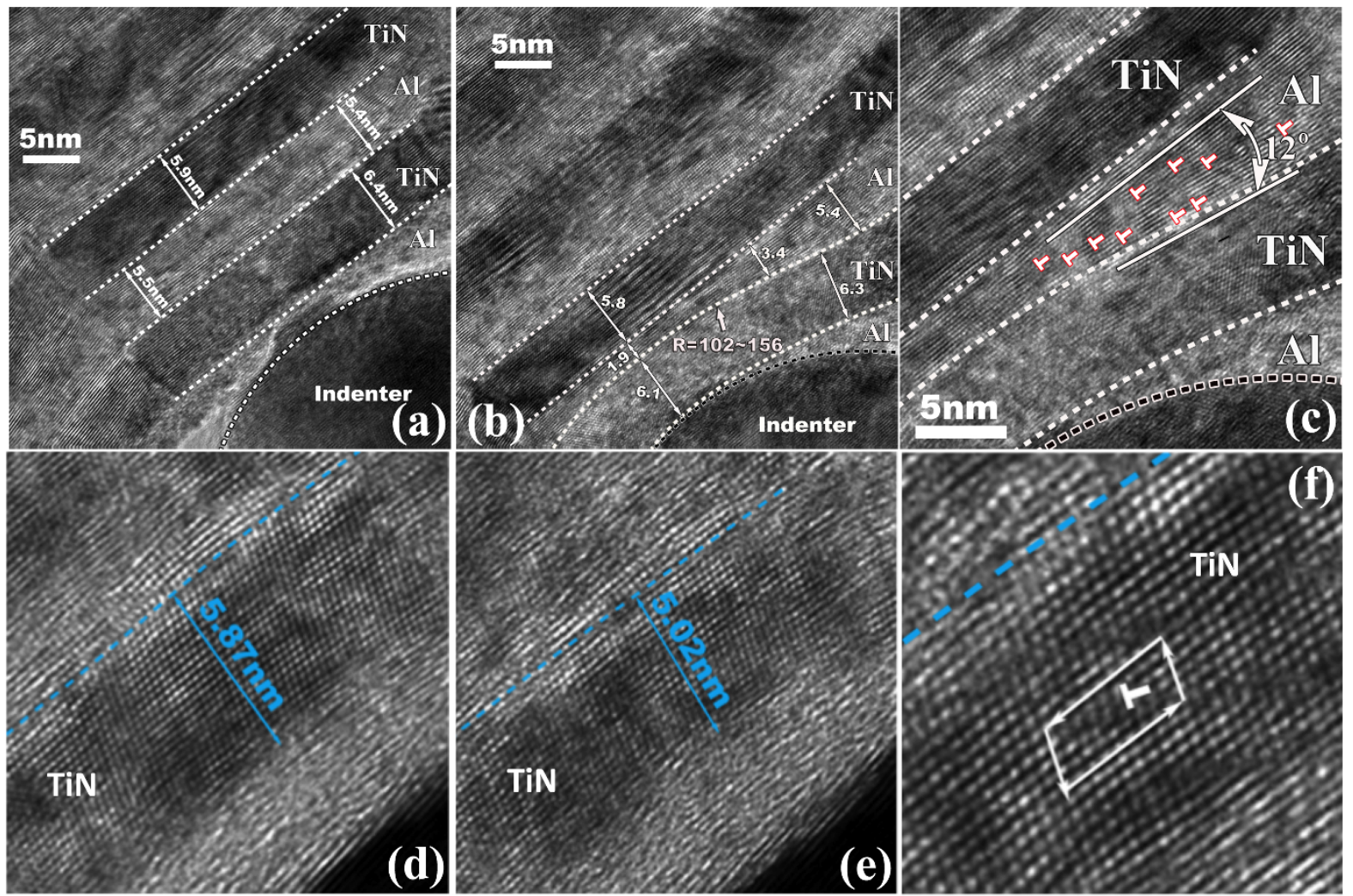

Figure $4 \mid$ HRTEM images of the $5 \mathrm{~nm}$ Al-5 nm TiN multilayers during indentation. (a) and (b) show the thickness reduction in the second Al layer. (c) The interface between the $1^{\text {st }}$ TiN layer and the $2^{\text {nd }}$ Al layer, showing a tilt boundary associated with the accumulation of dislocations in the Al layer and at the interface. (d) and (e) The thickness reduction in the first TiN layer from $5.87 \mathrm{~nm}$ to $5.02 \mathrm{~nm}$, and (f) a lattice dislocation characterized by the Burgers circuit in the TiN layer. 

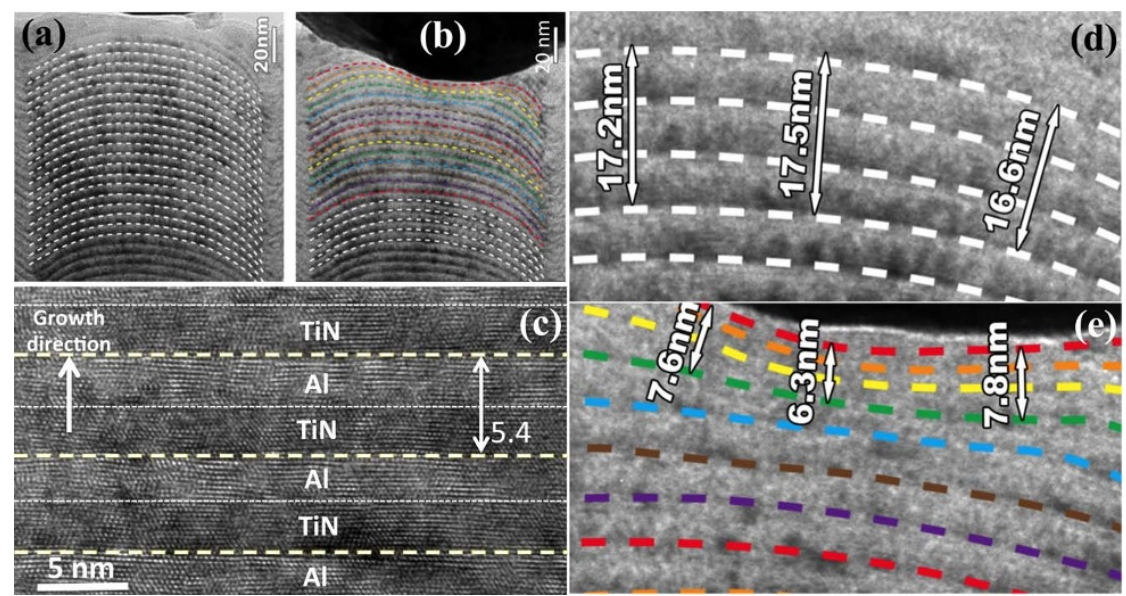

Figure 5 Figure 5.(a) and (b) TEM images of the $2.7 \mathrm{~nm} \mathrm{Al-2.7} \mathrm{nm}$ TiN multilayer before and during indentation. The dashed lines indicate the bi-layer thickness, as identified to be the Al-TiN interfaces in (c). (d) and (e) The thickness reduction in the first three bilayers from $17.5 \mathrm{~nm}$ to $6.3 \mathrm{~nm}$.

arrays produces a tensile residual stress $\sigma_{\text {ceramic }}^{\text {dislocations }}$ in the ceramic layers $^{20}$.

The increase of the tensile residual stress $\sigma_{\text {ceramic }}^{\text {dislocations }}$ could lead to Mode I fracture in the ceramic layers. On the other hand, the tensile residual stress also adds on to the resolved shear stress on the glide plane in the ceramic layer. In addition, the interaction between the deposited dislocations in the two adjacent interfaces also contributes to the resolved shear stress in the ceramic layer. Thus the net resolved shear stress in the ceramic layer is composed of three components, the contribution from the residual stress $\sigma_{\text {ceramic }}^{\text {dislocations }}$ due to the plastic incompatibility, the interaction force $\tau^{\text {interaction }}$ between the deposited dislocations in the adjacent interfaces, and the applied stress $\sigma^{\text {Applied }}$, and can be written as $\tau_{\text {ceramic }}^{\text {RSS }}=\tau^{\text {Interaction }}+m\left(\sigma_{\text {ceramic }}^{\text {dislocations }}+\sigma^{\text {Applied }}\right)$, where $m$ is the Schmid factor. According to the elastic mechanics and the dislocation theory ${ }^{27}, \tau^{\text {interaction }}$ increases with decreasing the ceramic layer thickness, and becomes a significant contributor when the layer thickness is $<\approx 10 \mathrm{~b}$ where $\mathrm{b}$ is the magnitude of Burgers vector. $\sigma_{\text {ceramic }}^{\text {dislocations }}$ increases with increasing density of the deposited dislocations at the interfaces. Under compression, the maximum applied stress $\sigma^{\text {Applied }}$ normal to the interface is determined by the confined layer slip stress in the metal layer, because the stress in the TiN layer is much higher than that in the Al layer. According to "smaller is stronger"28, decreasing the metal layer thickness can effectively increase the strength of the metal layer, in turn enhance the maximum applied stress. In addition, the maximum applied stress increases with plastic deformation due to the strain hardening ${ }^{20}$. With the high applied stress and the strong interaction force in the fine-layered composites, ceramics can plastically co-deform with the metal layer. Thus, ceramic plasticity in metal-ceramic multilayers is favored at low thickness of both the ceramic layer and the metal layer. This is consistent with our experiments.

\section{Methods}

Thin multilayers composed of alternating $\mathrm{Al}$ and $\mathrm{TiN}$ individual layers were deposited at room temperature using direct current $(\mathrm{dc})$ magnetron sputtering on Si substrates (with a top layer of amorphous $\mathrm{SiO}_{2}$ ) with equal volume fractions of $\mathrm{Al}$ : $\mathrm{TiN}=$ $1: 1$. The film thickness of all samples is around $2 \mu \mathrm{m}$. TEM samples were prepared by mechanical polishing to a final thickness of $20-30 \mu \mathrm{m}$ with diamond lapping film, followed by ion-milling using a Gatan ${ }^{\mathrm{TM}}$ PIPS ${ }^{\circledR}$ instrument operating at $3-5 \mathrm{kV}$. Our indentation experiments were conducted inside a FEI Tecnai F30 field emission gun transmission electron microscope equipped with a Nanofactory TEM-STM system. The TEM was operated at $300 \mathrm{kV}$, with a point-to-point resolution around $0.2 \mathrm{~nm}$. The TEM foils were then attached to a piezo-operated scanning tunneling microscope (STM) probe with silver paint, which served as one end of a Nanofactory TEM-STM platform. An etched W tip was the other end of platform. The STM probe with the W tip was compressed onto the TEM foil. We recorded the videos during indentation by a CCD (charge-coupled device) camera at 3 frames per second. The commercial software Abaqus 6.12 was used to perform the FEM simulation. The FEM model was generated according to the geometry of the sample (see Supplementary Figure S4). The model was finely meshed with the element type of CPS4 (15456 elements. The stress field was solved under the displacement boundary. The final geometry of the deformed TiN layer is achieved by gradually deforming within $\sim 50$ steps.

1. Abadias, G., Dub, S. \& Shmegera, R. Nanoindentation hardness and structure of ion beam sputtered TiN, W and TiN/W multilayer hard coatings. Surf. Coat. Technol. 200, 6538 (2006).

2. Salehinia, I. et al. Plastic deformation of metal/ceramic nanolayered composites. JOM. 66(10), 2078 (2014).

3. Sinnott, S. B. \& Dickey, E. C. Ceramic/metal interface structures and their relationship to atomic- and meso-scale properties. Mater. Sci. Eng. R Rep. 43, 1 (2003).

4. Kingery, W. D., Bowen, H. K. \& Uhlmann, D. R. Introduction to Ceramics $2^{\text {nd }}$ edn. (Wiley, 1975).

5. Toth, L. E. Transition Metal Carbides and Nitrides (Academic Press, 1971).

6. Oden, M., Ljungcrantz, H. \& Hultman, L. Characterization of the induced plastic zone in a single crystal $\mathrm{TiN}(001)$ film by nanoindentation and transmission electron microscopy. J. Mater. Res.12, 2134 (1997).

7. Minor, A. M. et al. In situ nanoindentation of epitaxial TiN/MgO (001) in a transmission electron microscope. J. Elec. Mater. 32, 1023 (2003).

8. Embury, J. D. \& Hirth, J. P. On dislocation storage and the mechanical response of fine scale microstructures. Acta Metall. Mater. 42, 2051 (1994).

9. Singh, D. R. P. et al. Micropillar compression of $\mathrm{Al} / \mathrm{SiC}$ nanolaminates. Acta Mater. 58, 6628 (2010).

10. Deng, X. et al. Mechanical Behavior of Multilayered Nanoscale Metal-Ceramic Composites. Adv. Eng. Mater. 7, 1099 (2005).

11. Mook, W. M. Indentation fracture response of Al-TiN nanolaminates. Mater. Res. Lett. 1, 102 (2013).

12. Bhattacharyya, D. et al. A transmission electron microscopy study of the deformation behavior underneath nanoindents in nanoscale Al-TiN multilayered composites. Philos. Mag. 90, 1711 (2010)

13. Barnett, S. A. \& Madan, A. Hardness and stability of metal-nitride nanoscale multilayers. Scr. Mater. 50, 739 (2004).

14. Salehinia, I. et al. Molecular dynamics simulations of plastic deformation in $\mathrm{Nb} /$ NbC multilayers. Int. J. Plast. 59, 119 (2014).

15. Dück, A. et al. Ti/TiN multilayer coatings: deposition technique, characterization and mechanical properties. Surf. Coat. Technol. 142-144, 579 (2001).

16. Deng, X. et al. Nanoindentation of nanolayered metal-ceramic composites. J. Mater. Eng. Perform. 14, 417 (2005).

17. Sun, P. L. et al. Characterization of nanoindentation damage in metal/ceramic nanolaminate composites by Transmission Electron Microscopy (TEM). Mater Sci. Eng. A 527, 2985 (2010).

18. Tang, G. et al. Indentation analysis of metal-ceramic multilayers at the nanoscale: numerical analysis and experimental verification. Acta Mater. 58, 2033 (2010).

19. Bhattacharyya, D. et al. Compressive flow behavior of Al-TiN multilayers at nanometer scale layer thickness. Acta Mater. 59, 3804 (2011).

20. Wang, J. \& Misra, A. Strain hardening in nanolayered thin films. Curr. Opin. Solid State Mater. Sci. 18, 19 (2014).

21. Wang, J. et al. Dislocation structures of $\Sigma 3\{112\}$ twin boundaries in face centered cubic metals. Appl. Phys. Lett. 95, 021908 (2009).

22. Wang, J. et al. Detwinning mechanisms for growth twins in face-centered cubic metals. Acta Mater. 58, 2262 (2010).

23. Yadav, S. K. et al. First-principles density functional theory study of generalized stacking faults in TiN and MgO. Philos. Mag. 94, 464 (2014). 
24. Yadav, S. K. et al., Core structure and Peierls stress of edge and screw dislocations in TiN: A density functional theory study. Acta Mater 74, 268 (2014).

25. Wang, J., Misra, A. \& Hirth, J. P. Shear response of $\Sigma 3\{112\}$ twin boundaries in face-centered-cubic metals. Phys. Rev. B 83, 064106 (2011).

26. Bufford, D. et al. In situ nanoindentation study on plasticity and work hardening in aluminium with incoherent twin boundaries. Nat. Commun. 5, 4864 (2014)

27. Hirth, J. P. \& Lothe, J. Theory of Dislocaitons (Krieger Publishing Company, Malabar, FL, 1992)

28. Uchic, M. D. et al. Sample dimensions influence strength and crystal plasticity. Science 305, 986 (2004).

\section{Acknowledgments}

This work was supported by the US Department of Energy, Office of Science, Office of Basic Energy Sciences. This work was performed, in part, at the Center for Integrated

Nanotechnologies, an Office of Science User Facility operated for the U.S. Department of Energy (DOE) Office of Science. LANL is operated by Los Alamos National Security, LLC, for the National Nuclear Security Administration of the U.S. Department of Energy under Contract No. DE-AC52-06NA25396.

\section{Author contributions}

N.L. performed in situ TEM indentation testing and data analysis. H.W. performed FEM analysis. A.M. and J.W. conceived this study. J.W. and N.L. prepared the first version of this manuscript. All authors participated the discussion and revision of this manuscript.

\section{Additional information}

Supplementary Information accompanies this paper at http://www.nature.com/ scientificreports

Competing financial interests: The authors declare no competing financial interests. How to cite this article: Li, N., Wang, H., Misra, A. \& Wang, J. In situ Nanoindentation Study of Plastic Co-deformation in Al-TiN Nanocomposites. Sci. Rep. 4, 6633; DOI:10.1038/srep06633 (2014)

This work is licensed under a Creative Commons Attribution-NonCommercialNoDerivs 4.0 International License. The images or other third party material in this article are included in the article's Creative Commons license, unless indicated otherwise in the credit line; if the material is not included under the Creative Commons license, users will need to obtain permission from the license holder in order to reproduce the material. To view a copy of this license, visit http:// creativecommons.org/licenses/by-nc-nd/4.0/ 